\title{
Perturbative QCD Signatures of Hybrid Hadrons \\ in Electroproduction at High $\boldsymbol{Q}^{2}$
}

\author{
Carl E. Carlson \\ Institute for Nuclear Theory, University of Washington \\ $H N-12$, Seattle, Washington 98195, USA \\ and \\ Physics Department, College of William and Mary \\ Williamsburg, Virginia 23185, USA \\ Nimai C. Mukhopadhyay \\ Institute for Nuclear Theory, University of Washington \\ $H N-12$, Seattle, Washington 98195, USA \\ and \\ Physics Department, Rensselaer Polytechnic Institute \\ Troy, New York 12180-3590, USA
}

\section{PREPARED FOR THE U.S. DEPARTMENT OF ENERGY UNDER GRANT DE-FG06-90ER40561}

This report was prepared as an account of work sponsored by the United States Government. Neither the United States nor any agency thereof, nor any of their employees, makes any warranty, express or implied, or assumes any legal liability or responsibility for the accuracy, completeness, or usefulness of any information, apparatus, product, or process disclosed, or represents that its use would not infringe privately owned rights. Reference herein to any specific commercial product, process, or service by trade name, mark, manufacturer, or otherwise, does not necessarily constitute or imply its endorsement, recommendation, or favorin $n_{E}$ by the United States Government or any agency thereof. The views and opinions of autiars expressed herein do not necessarily state or reflect those of the United States Government or any agency thereof.

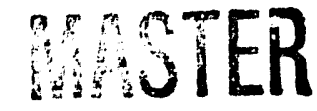


(1) Physics Department, College of William and Mary, Williamsburg, VA 23185

(2) Phy'sics Department, Rensselaer Polytechnic Institute, Troy, NY, 12180-3590

(3) Institute for Nuclear Theory, University of Washington, Scattle, WA 98195

\section{Abstract}

In the perturbative domain of quantum chromodynamics $(Q C D)$, transverse electroproduction of hybrid baryons is small. Their longitudinal electroproduction has size and scaling behavior like normal baryons. Thus decp inelastic scattering has hybrid resonance peak to background ratio small for the transverse structure function but normal size and constant for the longitudinal one. This signature can test if the Roper resonance is a hybrid. Related high momentum transfer signatures may clarify the structure of possible non-standard states such as $\Lambda(1405)$, $f_{0}(975)$, or $a_{0}(980)$. 
A hybrid hadron is one whose lowest significant Fock component contains a gluon. Such a gluon is often called a "valence gluon." States with valence gluons, viz, hybrid mesons [1], hybrid baryons [2], or glueballs [3], ought to exist, if QCD is the correct theory of strong interactions. A definite find of a hybrid or glueball would be very important in understanding the strong interaction region of QCD. A problem in isolating states with valence gluons is that they mix with ordinary hadron states. Thus, a hybrid hadron may have a normal three-quark (quark-antiquark) Fock component, albeit with smaller probability.

There has been a suggcstion that the Roper resonance N(1440) is a hybrid [4]. The idea explains why the Roper mass is unexpectedly light [5] and suggests that the nucleon to Roper electromagnetic transition is small $[G]$ for low $Q^{2}$ photons.

This paper studies, for the Roper and and for other hadrons, how to distinguish a hadron that is a hybrid or a molecular configuration from one that is a basic three-quark or quarkantiquark state, using the behavior of its production rate at high momentum transfer.

Spotlighting une case, we show that hybrid baryon electroproduction will fall relative to background at high $Q^{2}$. This in contrast to $q^{3}$ baryons, barring what one can call accidents of the wave function [7], whose resonance peak to background ratio is nearly constant $[8,9]$ as $Q^{2}$ changes. The results follow from an application of $Q C D$ counting rules [10] for the asymptotic bchavior of the form factor for a given hadron Fock component, and of those for the background. The $q^{3}$ Fock component normally has a form factor that gives an electroproduction rate falling with the same power of $Q^{2}$ as the background. However, for a hybrid the main $q^{3} G$ component leads to an elestroproduction rate falling faster by a factor $O\left(1 / Q^{2}\right)$.

We first attempt an estimate of hybrid baryon electroproduction rates. Let $\alpha^{2}$ and $1-\alpha^{2}$ be the relative probability of the normal baryonic component and pure hybrid component respectively, and take at $Q^{2}=0$ the contribution of each component to the hybrid form factor as proportional to its probability. Then, using dipole form factor mass scale, and omixing factors of $\left(\log Q^{2}\right)$, 


$$
\begin{aligned}
& G_{+}\left(Q^{2}\right)=\text { const. } \times\left\{\frac{\alpha^{2}}{\left(1+Q^{2} / 0.71 G e V^{2}\right)^{2}}+\frac{1-\alpha^{2}}{\left(1+Q^{2} / 0.71 G e V^{2}\right)^{3}}\right\}\left[Q^{2}+\left(m_{R}-m_{N}\right)^{2}\right]^{1 / 2}, \\
& G_{Q}\left(Q^{2}\right)=\text { const. } \times \frac{1}{\left(1+Q^{2} / 0.71 G e V^{2}\right)^{2}} .
\end{aligned}
$$

Here, the helicity amplitudes or form factors are $G_{+}, G_{0}$, and $G_{-} ;$when the nucleon has positive helicity, the subscript is the virtual photon helicity in the $\gamma^{*} N \rightarrow R$ Breit frame. The transverse amplitude $G_{+}$has nominally the slowest asymptotic falloff. Up to a $Q$-independent factor, it is the same as $A_{1 / 2}$. For the elastic case, it is $Q / m_{N} \sqrt{2}$ times the magnetic form factor $G_{M}$ The square root in (1) gives the known kinematic zero for the $N \rightarrow 1 / 2^{+}$and $N \rightarrow$ $3 / 2+$ transitions [11], and also gives $G_{+}$the correct $1 / Q^{3}$ asymptotic falloff for a normal baryon [12]. A pure hybrid $G_{+}$falls like $1 / Q^{5}$, as is explained below. Relaxing the use of a dipole mass scale affects the estimated size of the contributions at finite $Q^{2}$, but not the general conclusions. The mass scales are determined by the sizes of the statcs and should not differ greatly from each other. The longitudinal form factor $G_{\circ}\left(G_{E}\right.$ for the elastic case) is show'n to fall asymptotically like $1 / Q^{4}$ for both normal and hybrid baryons (thus the " 1 " in the numerator is $\left.a^{2}+\left(1-\alpha^{2}\right)\right)$ [13].

A normal non-hybrid baryon has $\alpha \approx 1$ and has roughly a constant 1:1 resonance peak/background ratio in $v W_{2}$. Asymptotically, the resonance contribution to $v W_{2}$ is

$$
v W_{2}^{\text {res }} \propto 2 G_{0}^{2}+G_{+}^{2}+G_{-}^{2}
$$

Hence -- taking this $1: 1$ ratio as standard for an ordinary baryon -- we have at $x_{R}$, referring to the location of a resonance peak, a ratio 


$$
\begin{gathered}
R=\frac{v W_{2}^{r c s}\left(x_{R}\right)}{v W_{2}^{\text {bkgd }}\left(x_{R}\right)} \approx \alpha^{4}+\frac{2 \alpha^{2}\left(1-\alpha^{2}\right)}{\left(1+Q^{2} / 0.71 G e V^{2}\right)}+\frac{\left(1-\alpha^{2}\right)^{2}}{\left(1+Q^{2} / 0.71 G e V^{2}\right)^{2}} \\
+o\left(1 / Q^{2}\right)
\end{gathered}
$$

The first three terms are transverse photon contributions, while the last term is a longitudinal contribution with the asymptotic form indicated.

Genuine hybrids are states with small $\alpha$. Thus, the resonance peak/background ratio for a hybrid that is seen at low $Q^{2}$ must fall with respect to background and be small at high $Q^{2}$. This is a necessary condition for a baryon to be a hybrid. With $\alpha=0$, the hybrid stands less than $4 \%$ above background in the transverse structure function when $Q^{2}$ is above $3 \mathrm{GeV}^{2}$, and is falling to zero. In $v W_{2}$, the longitudinal contribution will dominate, but will still be fairly small and falling like $1 / Q^{2}$. If some candidate hybrid is not seen at low $Q^{2}$, one must not expect it to surge to prominence at high $Q^{2}$.

We must stress that a small resonance peak/background ratio, while necessary, is not sufficient for a baryon resonance to be a hybrid. The resonance peak/background ratio can fall for a non-hybrid due to cancellations that make the leading form factor small. This may explain [7] the falling signal/background obscrved for the $\Delta(1232)$.

We now describe how to get the asymptotic behavior of the helicity amplitudes for hybrid elcctroproduction. The asymptotic $Q^{2}$ dependence of ordinary baryons is governed by diagrams like Fig. I(u). The rules for calculating the leading $Q$ dcpendence require a factor $Q$ for each quark line running through the diagram, a factor $1 / Q$ for each internal quark propagator, and a factor $1 / Q^{2}$ for each internal gluon propagator. Quark helicity is conserved to get the leading $Q$ dependence, and there are rules for the photon and gluon helicity [14]. For example, to obtain leading $Q$ dependence when one gluon attaches to a quark, it must be transverse; when two gluons or a gluon and a photon attach to a quark, one must be transverse and the other 
longitudinal. A violation of any helicity rule multiplies the asymptotic $Q$ behavior by at least one factor of $O(1 / Q)$. In ordinary baryons, $G_{+}, G_{0}$, and $G_{-}$go asymptotically like $1 / Q^{3}$, $1 / Q^{4}$, and $1 / Q^{5}$ respectively.

Hybrid electroproduction from a nucleon is illustrated in Fig. 1(b) using the nucleon $q^{3}$ Fock component or in Fig. 1 (c) using the nucleon $q^{3}$ G Fock component. Formalisms that allow a Fock space description of a hadron, including the light cone formalism, require the constituents to be on shell. Ilence the external gluon lines in Figs. 1(b,c) must be transverse. If all helicity rules are satisfied, the anplitudes in Fig. 1(b) are asymptotically $O\left(1 / Q^{4}\right)$ and those in Fig. 1(c) are $O\left(1 / Q^{5}\right)$. For $G_{+}$, it is not possible for any of the diagrams exemplified by Fig. 1(b) to satisfy the rules, so at least one more factor of $O(1 / Q)$ enters. For $G_{0}$, all helicity rules can be satisfied for the Fig. 1(b) amplitudes. In particular, quark helicity is conserved for the longitudinal hybrid production. Collecting results, the helicity amplitudes for ordinary baryon $\left(q^{3}\right)$ and hybrid $\left(q^{3} G\right)$ production satisfy

$$
\begin{gathered}
G_{+}\left(N \rightarrow q^{3}\right) \propto 1 / Q^{3} \\
G_{0}\left(N \rightarrow q^{3}\right) \propto 1 / Q^{4} \\
G_{+}\left(N \rightarrow q^{3} G\right) \propto 1 / Q^{5} \\
G_{0}\left(N \rightarrow q^{3} G\right) \propto 1 / Q^{4} .
\end{gathered}
$$

The longitudinal amplitude is asymutotically the largest for the hybrid and scales the same as for the ordinary hadron. Quark helicity conscrvation does not imply hadron helicity conservation if there are valence gluons. In longitudinal electroproduction [15] the hybrid will not fall relative to the background and both aspects of the BloJm-Gilman phenomenon [8] (resonances plus background avcraging to the scaling curve [16] and resonance peak/background ratio being constant [9]) should be valid.

Reduced production of hybrids relative to ordinary hadrons at high momentum tranfers also 
occurs elsewhere. Let us take some examples. In general, for $A+B \rightarrow C+D$, with c.m. encrgy $\sqrt{s}$ and momentum transfer $\sqrt{-t}$, both large and their ratio fixed,

$$
\frac{d \sigma}{d t} \propto s^{2-n_{A}-n_{B}-n_{C}-n_{D}}
$$

where $n_{f}$ is the number of elementary fields in particle $I$.

The $\Lambda(1405)$ is one state with possible unusual valence quark configurations. It may be a three quark state with one quark in the P-shell [17]. Alternatcly, it may be a $\vec{K} N$ bound state [18], minimally a five-constituent object. The true $\Lambda(1405)$ structure may be elucidated by the scaling of its cross section at high mometum transfer. Thus for associated photoproduction of the $\Lambda(1405)$, we get

$$
\begin{aligned}
& \frac{d \sigma}{d t}(\gamma N \rightarrow K \Lambda(1405)) \propto s^{-7}, \quad \text { if } \Lambda(1405)=q^{3} . \\
& \frac{d \sigma}{d t}(\gamma N \rightarrow K \Lambda(1405)) \times s^{-9}, \quad \text { if } \Lambda(1405)=\bar{K} N .
\end{aligned}
$$

Another prospect is $\Lambda(1405)$ production by a charge curremt weak intcraction. The leading leptoproduction form factor for a $\bar{K} N$ state would fall faster than for a $q^{3}$ state by $O\left(1 / Q^{2}\right)$.

Evidence, from spectroscopy and decays suggest the $f_{0}(975)$ and $a_{0}(980)$ are $q^{2} \bar{q}^{2}$ states [19-21]. Electromagnetic reactions can provide further evidence, as in the sequence

$$
\begin{array}{r}
\frac{d \sigma}{d t}\left(\gamma p \rightarrow \pi^{+} n\right) \propto s^{-7}, \\
\frac{d \sigma}{d t}(\gamma p \rightarrow(q \bar{q} G) N) \propto s^{-8}, \\
\frac{d \sigma}{d t}\left(\gamma p \rightarrow\left(q^{2} \bar{q}^{2}\right) N\right) \propto s^{-9} .
\end{array}
$$


Hybrid or molecular meson scaling behavior is also distinct from that of two-gluon glueballs, which have the same scaling behavior as $q \bar{q}$ mesons. An $O(1 / s)$ suppression does apply to three-gluon glueballs.

We now return to the suggestion that the Roper is a hybrid [4]. This agrees with the nonappearance of the Roper in high momentum transfer electroproduction, in contrast to the 1530 and $1688 \mathrm{MeV}$ resonance bumps that are clearly visible at the same momentum transfers. Earlier data analyses [22] showed a bump at about the Roper mass that grew prominent at $6 \mathrm{GeV}^{2}$, the highest $Q^{2}$ then shown, but it was not visible below $3 \mathrm{GeV}^{2}$. The most recent data [23] has smaller error bars in the few $\mathrm{GeV}^{2}$ region and extends to $21 \mathrm{GeV}^{2}$ and finds no Roper electroproduction.

Our work does not treat low $Q^{2}$ electroproduction, which is in the non-perturbative $Q C D$ domain. There are models where the Roper is a $2 S$ radial excitation of the nucleon [24] as well as models where it is a hybrid [4]. At high momentum transfers, results based on nonrelativistic wave functions or constituent quark models will not apply. If the Roper is a hybrid, its electroproduction rate remains small asymptotically, whereas if it is a $q^{3}$ state we have no reason for a small electroproduction rate at high $Q^{2}$. Further, if the Roper is a hybrid and its $P_{11}(1710)$ counterpart is a $q^{3}$ state, that difference should be reflected in their high $Q^{2}$ electroproductionfochavior.

One low $Q^{2}$ SU(6) result is the Moorhouse-Barnes-Close (MBC) selection rule [25], which forbids transitions from the proton, bur not the neutron, to states with quark spin-SU(3) configuration ${ }^{48}$. The hybrid Roper can $[4,6]$ overcome this as ${ }^{4} 8$ and ${ }^{2} 8$ states may mix. At high $Q^{2}$, short distance wave functions are relevant and spin dependent forces perturb them drastically from the SU(6) form, as in the Chernyak-Zhitnitsky example (26]. So, even if the MBC rule applics for photoproduction, one should not expect a high $Q^{2}$ suppression of electroproduction for this reason, nor should there be a difference between proton and neutron 
targets.

To summarize: the hybrid hypothesis for the Roper has a perturbative QCD signal. For a pure hybrid baryon, the transverse form factor falls asymptotically $O\left(1 / Q^{2}\right)$ faster than what is expected for a normal $q^{3}$ baryon. The longitudinal form factor of a hybrid baryon, on the other hand, falls at the same rate as a $q^{3}$ baryon. Hence, in electroproduction a pure hybrid falls relative to background by $O\left(1 / Q^{4}\right)$ in the transverse structure function and $O\left(1 / Q^{2}\right)$ in $v W_{2}$.

For a hybrid with some mixture of $\mathrm{q}^{3}$ state, there is a small part of the production rate that is constant with respect to background, in addition to another initially larger part that falls like $O\left(1 / Q^{2}\right)$ with respect to background. Though this resembles $\Delta(1232)$ electroproduction, the underlying reasons for the two are very different. In the latter there is wave function accident. Barring that for the Roper, we have a useful rule: a delta-like Roper is a hybrid. The exception could be checked in other exclusive processes.

Similar considerations apply to other high momentum transfer production processes for hybrids or molecular states. Thus, the scaling of the electromagnetic production rates for $\Lambda(1405), a_{0}(980)$, or $f_{0}(975)$ is different depending on whether they are molecular configurations or $q^{3}$ or $\overline{q q}$ states.

What momentum transfer is sufficient for asymptotic scaling to be seen? For resonance electroproduction, the nucieon, the $1520 \mathrm{MeV}$ bump, and the $1688 \mathrm{MeV}$ bump appear to scale as expected from perurbative $Q C D$, starting at $Q^{2}$ of a few $\mathrm{GeV}^{2}$ [23]. Hence CEBAF energies may suffice to see the transition from non-perturbative to perturbative electroproduction of hybrid hadrons.

We thank V. Burkert, B. Jennings, and Z. Li for useful remarks. We are grateful to E. Henley, N. Isgur, G. Karl, and G.E. Miller for the hospitality of the Institute for Nuclear Theory in Seattle. CEC is supported in part by the NSF (Grant \#PIYY-9112173) and NCM by the U.S. Department of Encrgy (Grant \#DE-FG02-88ER47048-A.003). CEC also thanks NATO for a collaborative travel grant. 


\section{REFERENCES AND FOOTNOTES}

[1] M. Chanowitz and S. Sharpe, Nucl. Phys. B222, 211 (1983); T. Barnes, F.E. Close, and F. deViron, Nucl. Phys. B224, 241 (1983); M. Flensburg, C. Peterson, and L. Sköld, Z. Phys. C 22, 293 (1984).

[2] H. Hogaasen and J. Wroldsen, Phys. Lett. 116B, 369 (1982); T. Bamcs and F. Close, Phys. Lett. 123B, 89 (1983); I. Duck and E. Umland, Phys. Lett. 128B, 221 (1983); E. Golowich, E. Hạq, and G. Karl, Phys. Rev. D 28, 160 (1983); C.E. Carlson and T.H. Hansson, Phys. Lett. 128B, 95 (1983).

[3] S. Meshkov, Proceedings of the Seventh International Confrerence on Experimental Meson Spectroscopy, Brookhaven, NY, 1983, ed. S.J. Lindenbaum, p. 125 [American Institute of Physics, N.Y., 1984]; M. Chanowitz, Proceedings of the Twelrth International Confercnce on Particles and Nuclei, Boston, MA, 1990, Nucl. Phys. A527B, 61c (1991).

[4] Z. Li, University of Tennessce report UTK-91-05 (1991).

[5] S. Capstick and N. Isgur, Phys. Rev. D 34, 2809 (1986).

[6] Z. Li, V. Burkert, and Z. Li, University of Tennessee report UTK-91-07/CEBAF report (1991).

[7] C.E. Carlson and J.L. Poor, Phys. Rev, D 38, 2758 (1988); V.L. Chernyak, A.A. Ogloblin, I.R. Zhitnitsky, Yad. Fiz. 48, 1398 (1988) [Trans.: Sov. J. Nucl. Phys. 48, 889 (1988)]; G.R. Farrar, H. Zhang, A.A. Ogloblin, and I.R. Zhitnitsky, Nuci. Phys. B311, 585 (1989); J. Bonekamp, Bonn Report BONN-IR-89-43 (1989) and W. Pfeil, private communication.

[8] E.D. Bloom and F. Gilman, Phys. Rev. Lett. 25, 1140 (1970) and Phys. Rev. D 4, 2901 (1971).

[9] C.E. Carlson and N.C. Mukhopadhyay, Phys. Rev. D 41, 2343 (1990).

[10] S. J. Brodsky and G. Farrar, Phys. Rev. Letr. 31, 1153 (1973) and Phys. Rev. D 11, 1309 
(1975); V.A. Matveev, R.M. Muradyan, and A.N. Tavkhelidze, Lett. Nuovo Cim. 7, 719 (1973).

[11] J.D. Bjorken and J.D. Walecka, Ann. Phys. (N.Y.) 38, 35 (1966).

[12] C.E. Carlson, Phys. Rev. D 34, 2704 (1986).

[13] For $G_{0}$ there is a kinematic zero for the non-elastic case. Our form fits the data for the elastic case; for other cases it is a high $Q^{2}$ approximation.

[14] A.I. Vainshteir and V.I. Zakharov, Phys. Lett. 72B, 368 (1978); C.E. Carlson and F. Gross, Phys. Rev. Lett. 53, 127 (1984).

[15] Regarding analysis of Roper production up to $Q^{2}=1 \mathrm{GeV}^{2}$ see Ch. Gearhart, Z. Phys. C 4. 311 (1980) and G. Kroesen, Bonn Diploma Thesis - IR-83-03 (1983), reported in V. Burkert, Research Program at CEBAF, ed. F. Gross, p. 5-1 (CEBAF, Newport News, 1986).

[16] A. DeRújula, H. Georgi, and H.D. Politzcr, Ann. Phys. (N.Y.) 103, 315 (1977).

[17] T.A. DeGrand, Ann. Phys. (N.Y.) 101, 496 (1977); Y. Umino and F. Myhrer, Phys. Rev. D 39, 3391 (1989).

[18] R.H. Dalitz and S.F. Tuan, Ann. Phys. (N.Y.) 10, 307 (1960); E.A. Veit, B.K. Jennings, A.W. Thomas, and R.C. Barrett, Fhys. Rev. D 31, 1033 (1985); B.K. Jennings, Phys. Lett. B 176, 229 (1986).

[19] R.L. Jaffe, Phys. Rev. D 15, 267 and 281 (1977); J. Weinstein and N. Isgur, Phys. Rev. D 41, 2236 (1990).

[20] F. Iachello, N.C. Mukhopadhyay, and L. Zhang, Phys. Lett. B 256, 275 (1991) and Phys. Rev. D 44, 898 (1991).

[21] Particle Data Group, Phys. Lett. B 239. VII.164 (1991).

[22] V. Burkert, Research Program at CEBAF (II), ed. V. Burkert et al., p. 178 (CEBAF, Newport New', 1987).

[23] P. Sioler, Phy's. Ruv. Lett. 66, 1003 (1991) and Phys. Rev. D 44, 73 (1991). 
[24] H.J. Weber, Phys. Rev. C 41, 2783 (1990); M. Warns, H. Schröder, W. Pfeil, and H. Rollnik, Z. Phys. C 45, 627 (1990)

[25] R.G. Mrsorhouse, Phys. Rev. Lett. 16, 772 (1966); T. Barnes and F.E. Close, Phys. Lett. B123, 89 (1983) and B128 277 (1983).

[26] 1.D. King and C.T. Sachrajda, Nucl. Phys. B279, 785 (1987); I.R. Zhitnitsky, A.A. Ogloblin, and V.L. Chernyak, Yad. Fiz. 48, 841 (1988) [Trans.: Sov. J. Nucl. Phys. 48, 536 (1988)]; M. Gari and N. Stefanis, Phys. Lett. B 175, 462 (1986) and Phys. Rev. D 35, 1074 (1987).

\section{FIGURE CAPTION}

Fig. 1. Examples of lowest order Feynman diagrams for electroproduction of (a) a three quark baryon from a nucleon, (b) a pure hybrid $\left(q^{3} G\right)$ baryon from a nucleon, and (c) a pure hybrid baryon from the $q^{3} G$ Fock component of a nucleon. 


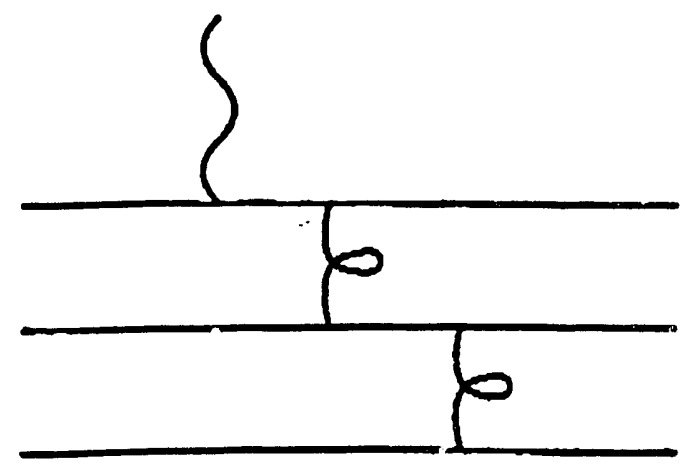

(a)
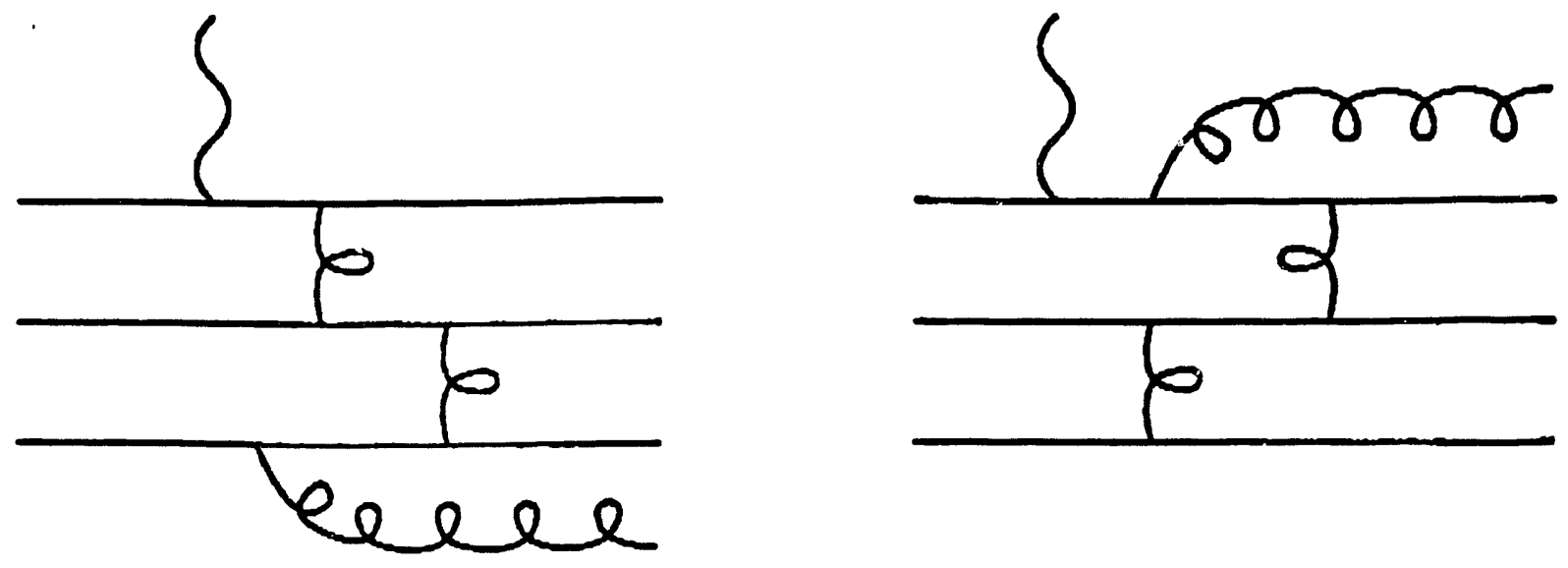

(b)
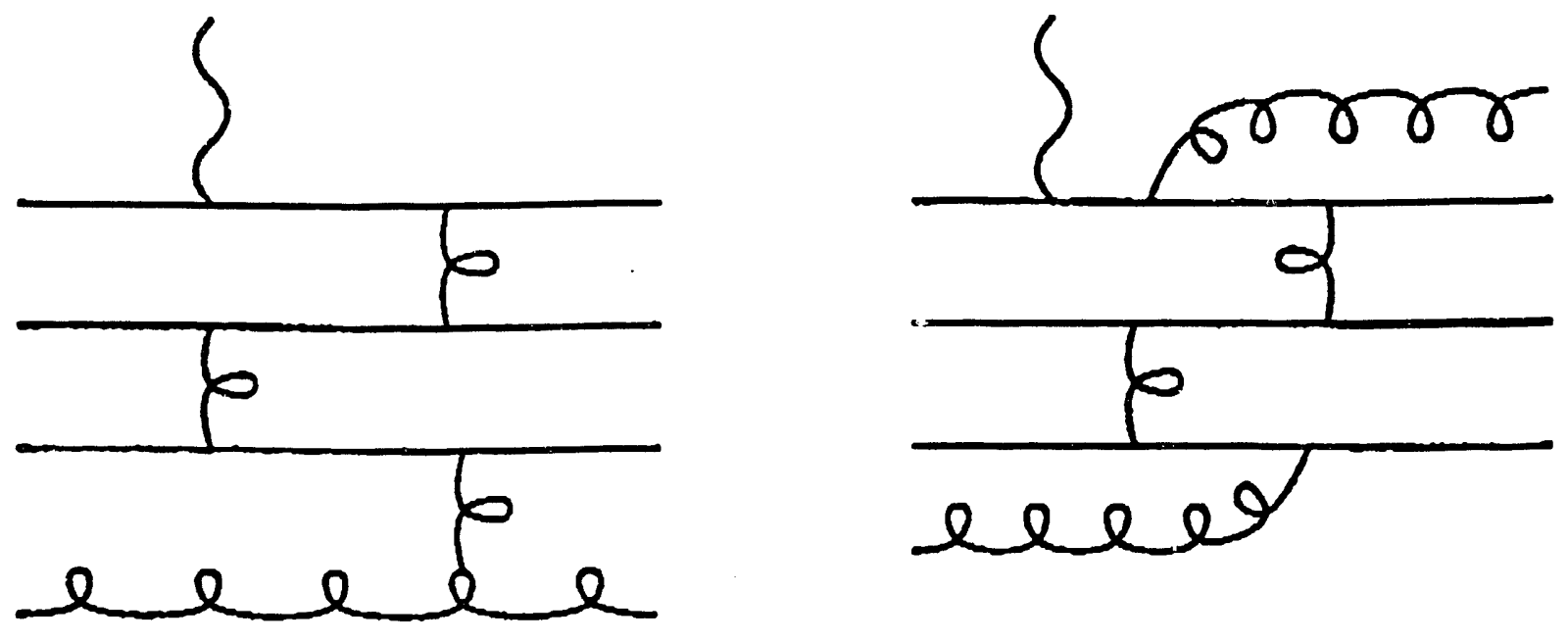

(c)

Fig. 1

page 12 

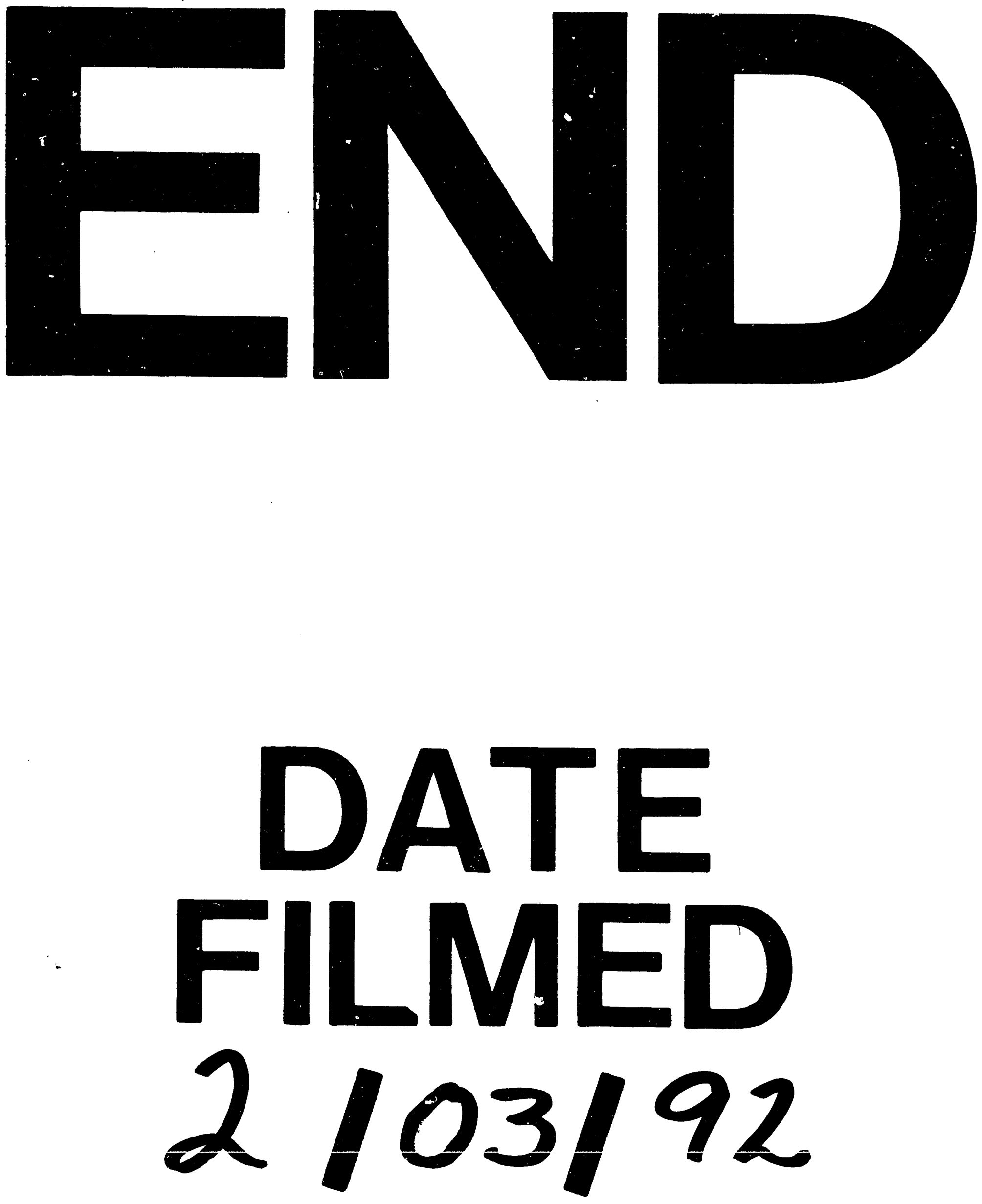
